\title{
Causalidad entre el gasto de Gobierno y el ingreso nacional: el caso de Bolivia
}

\section{Causality between Government Expenditure and National Income: The case of Bolivia}

Germán Molina Díaz* Marcelo Gantier Mita**

\section{Resumen}

El presente trabajo de investigación busca determinar la dirección de causalidad existente entre el gasto de Gobierno y el ingreso nacional en Bolivia durante el periodo 1990-2014. Con la finalidad de realizar una revisión del enunciado de la ley de Wagner y la teoría keynesiana, se procedió a la estimación de un Modelo de Vectores Autorregresivos y un Modelo de Vectores de Corrección de Error, logrando así analizar las relaciones de corto y largo plazo de las variables. Posteriormente se realizaron los Test de causalidad de Granger, para definir la dirección de causalidad existente entre ambas variables. Los resultados muestran que en el corto plazo se cumple la teoría keynesiana, para el período 1990-2014, en la economía boliviana. Por otro lado, analizando la relación de largo plazo se puede apreciar que se cumple la hipótesis de la neutralidad, es decir, las variables no se causan mutuamente, en el sentido de Granger.

Palabras clave: VAR, VEC, Causalidad de Granger, Ley de Wagner, Keynes.

\footnotetext{
Docente de la carrera de Economía de la Universidad Católica de Bolivia, Académico de número de la Academia Boliviana de Ciencias Económicas. Contacto: gmolinad@ucb.edu.bo

** Asistente de investigación del Instituto de Investigaciones Socio Económicas (IISEC). Contacto: marcelogantier@gmail.com
} 


\begin{abstract}
This research seeks to determine the direction of causality between government spending and national income in Bolivia during the period 1990-2014. In order to conduct a review of the statement of Wagner's law and Keynesian theory, we proceeded the estimation of a Vector Autoregressive Model and Vector Error Correction Model, achieving to analyze short and long term relationships of the variables. Later Granger causality test were performed to define the direction of causality between the two variables. The results show that in the short term Keynesian theory, for the period 1990-2014 is met in the Bolivian economy. On the other hand, analyzing the long term relationship, it can be seen that the hypothesis of neutrality is met, variables do not cause each other in the sense of Granger.
\end{abstract}

Keywords: VAR, VEC, Granger Causality, Wagner's Law, Keynes.

\title{
Clasificación/Classification JEL: H50, N40, N42
}

\section{Introducción}

La relación entre el gasto del Gobierno y el ingreso nacional es muy importante para el desarrollo de las políticas públicas (Richter y Paparas, 2012). Es por ello que el papel del sector público en la economía ha sido un tema de continuo debate entre los economistas durante décadas. Grandes pensadores de la ciencia económica dedicaron sus trabajos a explicar la relación entre Estado y mercado en sus diversas modalidades y en situaciones de crisis y auge económico, diferenciando economías desarrolladas, emergentes y en desarrollo.

Dicha relación ha sido estudiada ampliamente por los economistas a lo largo de la historia. El hacendista alemán Adolph Wagner (1890) observó que, a medida que el ingreso real per cápita de una nación se incrementaba, la proporción del gasto público sobre el total de los gastos también lo hacía. Esta relación más adelante pasó a denominarse la "Ley de Wagner". Según esta ley, si un país experimenta crecimiento económico a largo plazo su sector público será cada vez mayor.

Por otro lado, Keynes (1936) argumentó que el gasto público es un factor exógeno y un instrumento importante de la política fiscal que afecta al ingreso nacional (Ebaidalla, 2013). Afirmó que existen circunstancias en las cuales mantener un déficit fiscal es deseable para 
estimular la actividad económica. Es decir, el Estado tiene que intervenir a través del gasto público.

El alemán Adolph Wagner y el inglés John Maynard Keynes establecieron sus teorías sobre el gasto público y el crecimiento económico. No obstante, para el primero era una cuestión derivada de un crecimiento económico previo, mientras que el economista británico consideraba necesario un Estado más fuerte que ayudase al progreso de la economía mediante determinadas políticas públicas. La cuestión del gasto público y el crecimiento siempre ha permanecido entre los temas más importantes a tratar por economistas y organismos internacionales.

La pregunta de si el incremento del gasto de Gobierno es la causa del crecimiento económico o el crecimiento económico es la causa del incremento del gasto de Gobierno, es sumamente importante para los países en desarrollo, en los cuales el sector público contiene una proporción relativamente grande de los recursos económicos de la sociedad (Dogan y Tang, 2011). En Bolivia, durante los últimos años el tamaño del sector público, medido mediante el gasto gubernamental, se ha incrementado ${ }^{1}$. Si bien ambas variables presentan un comportamiento similar en términos de tendencia ${ }^{2}$, es importante analizar la relación de causalidad existente entre ambas variables. Para ello se procedió a estimar un modelo VAR y un modelo VEC para Bolivia, considerando el periodo 1990-2014, obteniendo así un análisis de corto y largo plazo. Posteriormente se realizaron los test de causalidad de Granger, para determinar la dirección de causalidad existente entre ambas variables.

El documento se estructura de la siguiente manera. La segunda sección desarrolla el marco teórico de la investigación, tomando en cuenta los estudios previos realizados en el área. La tercera sección se encarga de describir la metodología a ser utilizada en el documento. Posteriormente, la cuarta sección describe los principales resultados encontrados. Finalmente, la quinta sección resume las principales conclusiones a las que arriba el documento.

\section{Marco teórico}

En la literatura económica existe un gran número de documentos que analiza la relación entre el gasto de Gobierno y el ingreso de una nación. Si bien estos documentos utilizan 
distintas especificaciones, periodos de muestra y países, aún no se ha llegado a un consenso definitivo sobre la relación de ambas variables (Ebaidalla, 2013). Antes de analizar las principales líneas de investigación en el área y los resultados más importantes, se realizará una revisión de los fundamentos teóricos que respaldan a las relaciones propuestas por Wagner y Keynes, incluyendo a los clásicos.

\subsection{Los clásicos}

Los principios clásicos sobre finanzas públicas enfatizan en la utilización del endeudamiento únicamente en forma transitoria, es decir, que se debe emitir deuda en períodos de déficit y rescatar la deuda en períodos de superávit fiscal. Bajo esta percepción, el presupuesto público debería estar balanceado, excepto en los períodos transitorios, cuando se podría recurrir al endeudamiento. Los clásicos insistían en financiar los egresos con ingresos genuinos -fundamentalmente impuestos- porque veían en el endeudamiento público el peligro de una elevación de la tasa de interés, lo que a su vez contraería la inversión, retardando el crecimiento del producto.

El marco conceptual clásico se representa en forma simple:

$$
\frac{g-t}{b}=y
$$

Donde:

$g=$ Gastos del sector público respecto al PIB.

$t=$ Impuestos o ingresos del sector público respecto al PIB.

$b=$ Cambio del stock de la deuda respecto al PIB.

$y=$ Tasa de crecimiento del producto.

\subsection{Ley de Wagner}

El economista alemán Adolph Wagner descubrió a mediados del siglo XIX que "El tamaño del Gobierno tiende a crecer a medida que prospera el nivel de vida de los ciudadanos". Sin embargo, esta afirmación parece ser a priori una contradicción, debido a que los ciudadanos de un país desarrollado tenderán a depender menos del Estado, a diferencia de los habitantes de una nación emergente o en vías de desarrollo. Wagner señaló que "Al hacerse las sociedades 
más complejas, las necesidades de gasto público son mayores, y, por lo tanto, el gasto debe incrementarse". En países muy pobres, por el contrario, no existe una demanda de bienes públicos, simplemente porque falta casi todo.

Wagner formula una simple comprobación empírica a partir de sus estudios sobre el gasto público y no crítica el rol de los poderes públicos como agentes económicos determinantes en el nivel de renta de la población. De hecho, es considerado la figura central de lo que se llamó el "socialismo de Estado", y era muy crítico con el mercado y la libre competencia. Por lo tanto, sus ideas sobre el Estado se inclinaban a una mayor participación de éste en las actividades económicas, y en ningún momento alentó el principio de que el mejor Estado es el que no existe.

La ley de Wagner ha despertado sumo interés entre los economistas del sector público desde su redescubrimiento a través de la recopilación "Classics in Public Finance" (Musgrave y Peacock, 1967). Esta ley se basa en que el Estado asume de forma recurrente nuevas funciones en aras de hacer mejor su trabajo. De esta forma, sostiene Wagner, los poderes públicos satisfacen de forma creciente y de manera más completa las necesidades económicas de la población.

La relación funcional de la ley de Wagner es:

$$
G=f(P I B)
$$

Donde:

$G=$ Gasto público.

$P I B=$ Producto Interno Bruto.

\subsection{John Maynard Keynes}

En su obra "La teoría general de la ocupación, el interés y el dinero", Keynes, objetó el principio clásico de prudencia fiscal mediante presupuestos equilibrados y argumentó que existen circunstancias en las cuales mantener un déficit puede ser deseable para estimular la economía. La hipótesis de insuficiencia de demanda agregada indujo a una política activa de gasto público. Sin embargo, Keynes planteó que la política de gasto público tenía que ser de carácter transitorio, y además fue muy específico en que el gasto público no debía sustituir al 
gasto privado o las empresas públicas sustituir a las empresas privadas. Bajo este enfoque, la necesidad del gasto público surge por una supuesta insuficiencia del gasto privado y por lo tanto no correspondería que una empresa pública desplazara a una empresa privada.

El marco conceptual keynesiano objetó el principio de prudencia fiscal mediante "presupuestos balanceados". El argumento es que existen circunstancias en las cuales mantener un déficit puede ser deseable para estimular la actividad económica. Por lo tanto, cuando se registra que los gastos son mayores a los impuestos $(g>t)$ se recurre a la financiación del déficit vía endeudamiento con bonos $(b)$.

En las finanzas públicas es importante no confundir déficit fiscal con gasto público, y es fundamental señalar que quienes enfatizan que el gasto público per se, y no el déficit o su financiamiento, es la causa de la inflación, se refieren a que el gasto público tiene un efecto vía tasa de interés real. Es decir, afecta a los sectores productivos, que a su vez hacen disminuir el producto y, por lo tanto, se incrementa la tasa de inflación.

Otra fuente de financiamiento para cubrir el déficit fiscal es mediante dinero adicional a los bonos. Es decir, la base monetaria se puede expandir para otorgar fondos al gobierno o para aumentar el stock de activos netos (oro y divisas) del banco central. Formalmente lo descrito anteriormente se puede expresar de la siguiente manera:

$$
v n(g-t)=p
$$

Donde:

$v=$ Velocidad de circulación del agregado monetario.

$n=$ Multiplicador monetario.

$g=$ Gastos del sector público respecto al PIB.

$t=$ Impuestos o ingresos del sector público respecto al PIB

$p=$ Tasa de inflación.

Finalmente, es importante remarcar que la relación entre el gasto público y el ingreso nacional propuesta por Keynes, afirma que el gasto público es una variable exógena, por lo tanto, el producto interno bruto puede ser afectado por el mismo. Formalmente la relación de causalidad sería la siguiente: 


$$
Y=f(G)
$$

Donde:

$$
\begin{aligned}
& Y=\text { Producto interno bruto } \\
& G=\text { Gasto total del sector público }
\end{aligned}
$$

\subsection{Gasto público e ingreso nacional}

Existen cuatro tipos de dirección de causalidad entre el gasto público y el ingreso nacional (Magazzino, 2012). Las distintas hipótesis que subyacen a la dirección de causalidad vienen descritas a continuación:

i. Hipótesis de neutralidad: La hipótesis de neutralidad sostiene que no existe ningún tipo de relación entre el gasto público y el crecimiento económico. Bajo este enfoque, el Gobierno no tiene una influencia sobre el crecimiento económico, por lo tanto, no debería intervenir en la economía.

ii. Hipótesis wagneriana: Como se mencionó anteriormente, la hipótesis wagneriana sostiene que el crecimiento del producto tiene una relación de causalidad con el gasto público.

iii. Hipótesis keynesiana: Contraria a la anterior, la hipótesis keynesiana sostiene que la relación de causalidad ocurre de manera inversa, es decir, el gasto público tiene una relación de causalidad con el crecimiento del producto.

iv. Hipótesis de retroalimentación: A diferencia de las anteriores hipótesis de causalidad, la hipótesis de retroalimentación sostiene que la causalidad no ocurre de manera unidireccional, sino más bien, bidireccional. Es decir, el PIB tiene efecto sobre el gasto público y viceversa.

Uno de los estudios seminales en el campo del gasto público y el ingreso nacional fue el desarrollado por Aschauer (1989). El autor analiza la relación existente entre la productividad agregada y el stock y el flujo de las variables que conforman el gasto de Gobierno. Para ello, introduce el stock de capital público como un insumo más en la función de producción. Realizando esta incorporación, el autor demuestra que el stock de infraestructura tiene una 
influencia sobre la productividad total de los factores, remarcando así la importancia del gasto público en el crecimiento económico.

A partir de este documento, los trabajos relacionados con las leyes de Wagner y Keynes se han enfocado en analizar la relación de causalidad existente entre las variables de gasto público e ingreso nacional. Es importante mencionar que aún no se ha llegado a un consenso sobre la relación de causalidad que se produce entre ambas variables. Entre los trabajos más recientes que apoyan la Ley de Wagner se encuentra el de Magazzino (2012), que analiza la relación entre el gasto público y el PIB en la economía italiana. El autor realiza un análisis tanto de corto como de largo plazo mediante modelos econométricos VAR y VEC, respectivamente. Concluye que la Ley de Wagner se cumple en ambos análisis (de corto y largo plazo), demostrando así que el crecimiento del producto interno bruto precedería al gasto público.

De la misma manera, Comín, Díaz y Revuelta (2009) demuestran que la Ley de Wagner se cumple para Argentina, Brasil, España y México. Su análisis contempla los años de 1990 a 2000 y analizan las relaciones de corto y largo plazo. Los autores concluyen que en los cuatro países analizados el Producto Interno Bruto tendría relación de causalidad con el gasto público. Por su parte, Islam (2001), encuentra la misma relación en su análisis realizado para Estados Unidos durante el periodo 1929-1996.

Por otro lado, Ebaidalla (2013) demuestra que, para el caso de Sudán, la relación de causalidad corresponde a la hipótesis keynesiana. Es decir, el gasto público antecedería al crecimiento económico. Al igual que los anteriores dos documentos, el autor analiza las relaciones de corto y largo plazo, demostrando que existe una influencia positiva del gasto de gobierno en el Producto Interno Bruto. Este resultado concuerda con el encontrado por Dogan y Tang (2011) en su análisis realizado para Filipinas.

La evidencia empírica también ha demostrado que la hipótesis de retroalimentación se cumple para algunas economías. Cheng y Lai (1997) demuestran que existe una causalidad bidireccional entre el crecimiento económico y el gasto público en Corea del Sur. Concluyen que, a causa de esta bidireccionalidad, se cumplen tanto la Ley de Wagner como la Ley de Keynes.

Entre los estudios realizados en Bolivia referidos al tema, es importante mencionar el trabajo desarrollado por Montero (2012). Con la utilización de un modelo de "Datos de Panel" en el periodo de 1989-2008, el autor demuestra que el Producto Interno Bruto departamental 
se encuentra negativamente relacionado con la inversión pública. Además remarca que esta relación no es estadísticamente significativa. Concluye que el hecho de que la inversión pública departamental se relacione negativamente con el PIB y no sea estadísticamente significativa se debe a que las inversiones públicas en Bolivia no buscan rentabilidad económica, sino más bien se enfocan en satisfacer ciertas necesidades de la población.

Por otra parte, Moscoso y Laserna (2012), mediante la utilización de un VAR estructural, encontraron que el gasto público tiene un efecto negativo y poco significativo en el producto. Entre las principales causas que los autores le atribuyen a este resultado se encuentra la posible existencia de una fuerte Equivalencia Ricardiana en la economía boliviana, donde los consumidores estarían anticipando una subida de impuestos a futuro debido a los incrementos del gasto público. Este resultado se encuentra en concordancia con el obtenido por Puig (2015), quien demuestra que el gasto público en Bolivia tiene un efecto multiplicador imperceptible y que carece de soporte estadístico.

\section{Metodología}

Para analizar la relación de corto y largo plazo existente entre el gasto de Gobierno y el ingreso nacional, se procedió a estimar un modelo de Vectores Autorregresivos (VAR) y un modelo de Vectores con Corrección de Error (VEC).

Los modelos econométricos de Vectores Autorregresivos (VAR) son modelos de series de tiempo multivariados, utilizados para el análisis macroeconómico originalmente por Sims durante la década de los 80 (Arias y Torres, 2004). Estos modelos son utilizados para describir el proceso generador de datos de un grupo de variables de series de tiempo. Debido a que todas las variables son tomadas como endógenas, estos modelos son capaces de caracterizar las interacciones simultáneas que se transmiten a lo largo de un determinado número de periodos entre un grupo de variables.

Una de las ventajas de estimar un modelo VAR consiste en el tipo de información que se obtiene del sistema de ecuaciones que se estima (Sims, 1980). A partir de las funciones de impulso-respuesta se puede analizar el signo, la intensidad y la persistencia que cada una de las innovaciones estocásticas tiene sobre las variables del modelo.

La estructura del sistema de Vectores Autorregresivos incorpora efectos de retroalimentación, ya que tanto las variables dependientes como las independientes pueden 
afectarse entre sí. Formalmente, el modelo de Vectores Autorregresivos de orden $n$ viene descrito de la siguiente manera (Enders, 2008):

$$
\left[\begin{array}{c}
x_{1 t} \\
x_{2 t} \\
\vdots \\
\vdots \\
x_{n t}
\end{array}\right]=\left[\begin{array}{c}
A_{10} \\
A_{20} \\
\vdots \\
\vdots \\
A_{n t}
\end{array}\right]+\left[\begin{array}{cccc}
A_{11}(L) & A_{12}(L) & \cdots & A_{1 m}(L) \\
A_{21}(L) & A_{22}(L) & \cdots & A_{2 m}(L) \\
\vdots & \vdots & \cdots & \vdots \\
\vdots & \vdots & \cdots & \vdots \\
A_{n 1}(L) & A_{n 2}(L) & \cdots & A_{n m}(L)
\end{array}\right]\left[\begin{array}{c}
x_{1 t-1} \\
x_{2 t-1} \\
\vdots \\
\vdots \\
x_{n t-1}
\end{array}\right]+\left[\begin{array}{c}
e_{1 t} \\
e_{2 t} \\
\vdots \\
\vdots \\
e_{n t}
\end{array}\right]
$$

Donde:

$x_{i t}=$ Variables endógenas.

$A_{i 0}=$ Parámetros que representan los términos de intercepto.

$A_{i j}(L)=$ Polinomios en el operador de rezago $(\mathrm{L})$.

$e_{i t}=$ Términos de perturbación.

Dado que en la parte derecha de la anterior ecuación solo aparecen los rezagos de las variables endógenas, no existe simultaneidad, por lo que la técnica de estimación de Mínimos Cuadrados Ordinarios es apropiada para llevar a cabo las estimaciones (McCandless et al., 2001). Por su parte, la elección del número de rezagos de cada una de las variables se realiza utilizando el test F, el Criterio de información de Akaike o el Criterio de Schwartz. El primer test contrasta la hipótesis nula de que las variables (rezagadas) adicionales no contienen información relevante como para mejorar los pronósticos de la variable de interés, y las dos últimas tratan de test que castigan los coeficientes extras (los correspondientes a las variables rezagadas adicionales), por lo que la especificación elegida corresponderá a aquél con el criterio más pequeño (Humérez y Jiménez, 1995).

Para analizar la relación de largo plazo de las variables, se procederá a analizar el test de cointegración multivariado de Johansen y Juselius (1990). Se puede especificar éste desde un modelo VAR de orden $k$, definido de la siguiente manera:

$$
y_{t}=A_{1} y_{t-1}+\ldots+A_{k} y_{t-k}+B x_{t}+e_{t}
$$


Donde $y_{t}$ es un vector $(n x 1)$ de variables no estacionarias $I(1), x_{t}$ es un vector que contiene los términos determinísticos, $A_{t}$ es una matriz de parámetros $(1 x n)$ y $e_{t}$ es un término de ruido blanco. Esta especificación del modelo VAR puede ser escrita en primera diferencia, para darnos el siguiente Modelo de Vectores con corrección de error (VEC) (Magazzino, 2012):

$$
\Delta y_{t}=\Pi \mathrm{y}_{\mathrm{t}-1}+\sum_{i=1}^{k-1} \Gamma_{i} \Delta y_{t-i}+B x_{t}
$$

Donde:

$$
\begin{gathered}
\Pi=\sum_{i=1}^{k} A_{i}-I \\
\Gamma_{i}=-\sum_{j=i+1}^{p} A_{j}
\end{gathered}
$$

El número de relaciones de cointegración se encuentra entre 0 y $n-1$, por lo tanto, el procedimiento de Johansen para testear la presencia de cointegración se enfoca en el rango de $\prod$. De verificarse la presencia de un vector de cointegración, se procederá a la estimación del modelo VEC para observar la relación de largo plazo de las variables.

\section{Resultados}

Para analizar la relación entre gasto de Gobierno e ingreso nacional, se recurrió a las cifras oficiales presentadas por el Instituto Nacional de Estadística. Las variables utilizadas son las siguientes: Gasto de Consumo Final de la Administración Pública (Gasto público), Producto Interno Bruto (PIB), Consumo de los Hogares, Inversión, Exportaciones e Importaciones, en frecuencia trimestral desde 1990 hasta 2014.

Previamente a la estimación del modelo VAR, se realizaron pruebas de raíz unitaria, con el fin de determinar el orden de integración necesario para lograr la estacionariedad de las variables. Los test de raíz unitaria demostraron que las variables endógenas en logaritmos presentan raíz unitaria; los resultados se encuentran descritos a continuación: 


\section{Cuadro 1 \\ Test de raíz unitaria}

\begin{tabular}{lccccc}
\hline & \multicolumn{4}{c}{ Test estadístico de Dickey-Fueller aumentado } \\
\cline { 2 - 5 } & \multicolumn{2}{c}{ Variables en nivel } & \multicolumn{2}{c}{ Primera diferencia } & Orden de \\
\cline { 2 - 5 } & Estadístico t & Prob. & Estadístico $t$ & Prob. & integración \\
\hline Ln PIB & 1,508 & 0,999 & $-1,389$ & 0.0001 & $\mathrm{I}(1)$ \\
\hline Ln Cons & 2,203 & 1,000 & $-1,150$ & 0.0001 & $\mathrm{I}(1)$ \\
\hline Ln Inv & 1,443 & 0,558 & $-1,438$ & 0.0001 & $\mathrm{I}(1)$ \\
\hline Ln Ggob & 1,649 & 0,999 & $-9,673$ & 0.0000 & $\mathrm{I}(1)$ \\
\hline Ln Exp & $-0,530$ & 0,880 & $-9,268$ & 0.0000 & $\mathrm{I}(1)$ \\
\hline Ln Imp & $-0,884$ & 0,789 & $-1,262$ & 0.0001 & $\mathrm{I}(1)$ \\
\hline
\end{tabular}

Fuente: Elaboración propia en base a datos del INE

Como podemos apreciar, no se rechaza la hipótesis nula de raíz unitaria en ninguna de las variables en nivel. Por lo tanto, las variables presentan raíz unitaria. Para poder elaborar el modelo de Vectores Autorregresivos (VAR), es necesario que las variables endógenas sean estacionarias. Es por ello que se procedió a diferenciarlas. La primera diferencia de las variables bastó para que todas éstas sean estacionarias.

Una vez que todas las variables demostraron ser estacionarias, se procedió a estimar el modelo de Vectores Autorregresivos con variables cointegradas de orden 1. Se determinó que el número de rezagos óptimo de las variables endógenas es 4.

El modelo de Vectores Autorregresivos estimado corresponde a la ecuación fundamental del PIB, medida desde el lado del gasto; se encuentra definido como²:

$$
Y_{t}=B(L) Y_{t}+e
$$

Las variables endógenas vienen determinadas por:

$g P I B_{t}=$ Tasa de crecimiento del Producto Interno Bruto.

$g$ Cons $_{t}=$ Tasa de crecimiento del consumo de los hogares.

$g \operatorname{In} v_{t}=$ Tasa de crecimiento de la inversión.

3 Los resultados del modelo VAR y las pruebas de estabilidad y auto-correlación serial se encuentran descritos en el anexo 3 y 4 respectivamente. 
$g G o b_{t}=$ Tasa de crecimiento del gasto de Gobierno.

$g E s p_{t}=$ Tasa de crecimiento de las exportaciones.

$g \operatorname{Imp} p_{t}=$ Tasa de crecimiento de las importaciones.

Es importante mencionar que, si bien todas las variables no parecen ser representativas guiándonos por el estadístico t, el mismo se ve afectado por la colinealidad endógena que presenta el modelo (Enders, 2008). Por lo tanto, se decidió mantener el modelo estimado con todas sus variables.

Los resultados del modelo VAR (ver Anexo 3) muestran que los signos de los rezagos del gasto que explican el comportamiento del PIB son negativos y poco significativos. Este resultado se encuentra en concordancia con los resultados obtenidos por Montero (2012), Moscoso y Laserna (2012) y Puig (2015).

Posteriormente se procedió a realizar el test de causalidad de Granger, para poder determinar el sentido de causalidad existente entre el PIB y el gasto público. De esta manera, se puede determinar cuál de las siguientes afirmaciones se cumple para el caso boliviano:

i. Cambios en el PIB causan cambios en el gasto público (ley de Wagner).

ii. Cambios en el gasto público causan cambios en el PIB (hipótesis keynesiana)

iii. El gasto público y el PIB no se encuentran relacionados. (hipótesis de neutralidad)

iv. Cambios en el PIB causan cambios en el gasto público y viceversa. (hipótesis de bidireccionalidad)

El objetivo del test de causalidad de Granger es especificar el sentido de la causalidad existente entre el gasto de Gobierno y el ingreso nacional. Para ello, se plantearon dos test.

El primero corresponde a la ley de Wagner. Este test considera como hipótesis nula que el Producto Interno Bruto no tiene efecto, en el sentido de Granger, en el gasto público. Por lo tanto, la hipótesis alterna afirma que el Producto Interno Bruto tiene efecto, en el sentido de Granger, en el gasto público.

El segundo test corresponde a la hipótesis keynesiana. La hipótesis nula afirma que el gasto público no tiene efecto, en el sentido de Granger, en el Producto Interno Bruto. Por otro lado, la hipótesis alterna afirma que el gasto público tiene efecto, en el sentido de Granger, en el Producto Interno Bruto. 
Para el primer test planteado, se obtuvieron los siguientes resultados:

Ho: El PIB no tiene efecto, en el sentido de Granger, en el gasto público.

$H a$ : El PIB tiene efecto, en el sentido de Granger, en el gasto público.

Test de causalidad de Granger

\begin{tabular}{cccc}
\hline \multicolumn{4}{c}{ Variable dependiente GGOB } \\
\hline Variables & Chi-2 & Df & Prob. \\
\hline GPIB & 2.9650260 & 4 & 0.5637 \\
\hline
\end{tabular}

Fuente: Elaboración propia en base a la los resultados del modelo VAR

Los resultados demuestran que, a un nivel de confianza del 95\%, no se rechaza la hipótesis nula $(\mathrm{Ho})$. Por lo tanto, podemos concluir que el PIB no tiene efecto, en el sentido de Granger, en el gasto público. En otras palabras, el PIB no tiene efectos sobre el gasto público. Por lo tanto, la ley de Wagner no se cumple para el caso boliviano.

Para el segundo test planteado, se obtuvieron los siguientes resultados:

Ho: El gasto público no tiene efecto, en el sentido de Granger, en el PIB.

$H a$ : El gasto público tiene efecto, en el sentido de Granger, en el PIB.

\begin{tabular}{cccc}
\multicolumn{4}{c}{ Cuadro 3 } \\
& Test de causalidad de Granger \\
\hline & Variable dependiente GPIB \\
\hline Variables & Chi-2 & df & Prob. \\
\hline GGOB & 7.919244 & 4 & 0.0946 \\
\hline
\end{tabular}

Fuente: Elaboración propia en base a la los resultados del modelo VAR.

Los resultados del segundo test demuestran que, a un nivel de confianza del $90 \%$, se rechaza la hipótesis nula $(\mathrm{Ho})$. En consecuencia, se acepta la hipótesis alternativa $(\mathrm{Ha})$, es decir, el gasto público tiene efecto, en el sentido de Granger, en el PIB. En síntesis, el gasto público tiene efectos sobre el PIB en el corto plazo. Por lo tanto, el modelo de vectores arutorregresivos nos muestra que la ley de Keynes se cumple para el caso boliviano.

Lo anterior se ve reflejado en la descomposición de varianza del modelo. Como se puede apreciar a continuación, aproximadamente el 29\% de las variaciones del PIB se encuentran explicadas por las variaciones en el gasto público. Por otro lado, solamente el 1.71\% de las 
variaciones del gasto público se encontrarían explicadas por las variaciones del PIB (ver Cuadro 5). Esto nos demuestra que el gasto público es mucho más relevante para el PIB que el PIB para el gasto público.

Cuadro 4

Descomposición de la varianza del PIB

\begin{tabular}{cccccccc}
\hline Periodo & Error Est. & D(GIMP) & D(GEXP) & D(GGOB) & D(GINV) & D(GCONS) & D(GPIB) \\
\hline 1 & 0.067313 & 4.112464 & 0.062197 & 28.98908 & 2.555337 & 21.20417 & 43.07675 \\
\hline 2 & 0.073486 & 3.378487 & 3.815149 & 32.14800 & 2.724287 & 22.69061 & 35.24346 \\
\hline 3 & 0.076135 & 4.549444 & 3.706921 & 32.50181 & 5.280038 & 20.93431 & 33.02747 \\
\hline 4 & 0.080178 & 4.624516 & 4.784025 & 32.04008 & 5.315632 & 21.29038 & 31.94537 \\
\hline 5 & 0.082874 & 6.421500 & 5.280249 & 30.30061 & 7.147154 & 20.63771 & 30.21278 \\
\hline 6 & 0.084662 & 7.220877 & 5.182930 & 30.10508 & 7.479368 & 20.39062 & 29.62112 \\
\hline 7 & 0.085458 & 7.280168 & 5.132558 & 29.81254 & 7.509452 & 20.76270 & 29.50258 \\
\hline 8 & 0.086073 & 7.251264 & 5.100535 & 29.72210 & 7.675370 & 20.94583 & 29.30489 \\
\hline 9 & 0.086340 & 7.231395 & 5.106601 & 29.64685 & 7.635872 & 21.12552 & 29.25376 \\
\hline 10 & 0.086773 & 7.378127 & 5.113210 & 29.50773 & 7.612261 & 21.20036 & 29.18832 \\
\hline 11 & 0.086934 & 7.511470 & 5.097554 & 29.46379 & 7.645264 & 21.18498 & 29.09694 \\
\hline 12 & 0.087125 & 7.529561 & 5.095339 & 29.45898 & 7.661901 & 21.19686 & 29.05736 \\
\hline 13 & 0.087239 & 7.512435 & 5.120852 & 29.38239 & 7.802573 & 21.17351 & 29.00824 \\
\hline 14 & 0.087295 & 7.506886 & 5.128012 & 29.31104 & 7.956741 & 21.15873 & 28.93859 \\
\hline 15 & 0.087336 & 7.529857 & 5.123998 & 29.29191 & 7.958463 & 21.14913 & 28.94664 \\
\hline
\end{tabular}

Ordenamiento de Cholesky: D(GIMP) D(GEXP) D(GGOB) D(GINV) D(GCONS) D(GPIB)

Fuente: Elaboración propia en base a la los resultados del modelo VAR 


\section{Cuadro 5}

Descomposición de la varianza del gasto público

\begin{tabular}{cccccccc}
\hline Periodo & Error Est & D(GIMP) & D(GEXP) & D(GGOB) & D(GINV) & D(GCONS) & D(GPIB) \\
\hline 1 & 0.067313 & 0.163375 & 0.030973 & 99.80565 & 0.000000 & 0.000000 & 0.000000 \\
\hline 2 & 0.073486 & 1.008625 & 0.080103 & 95.19938 & 1.272117 & 1.415303 & 1.024472 \\
\hline 3 & 0.076135 & 2.380398 & 0.714412 & 91.49168 & 2.355141 & 1.952936 & 1.105429 \\
\hline 4 & 0.080178 & 3.953875 & 1.643205 & 88.30880 & 2.488684 & 2.281416 & 1.324022 \\
\hline 5 & 0.082874 & 6.716802 & 1.632970 & 85.42962 & 2.723351 & 2.218571 & 1.278691 \\
\hline 6 & 0.084662 & 10.13136 & 1.613316 & 81.86242 & 2.634014 & 2.493813 & 1.265069 \\
\hline 7 & 0.085458 & 10.93651 & 1.582422 & 80.85941 & 2.603559 & 2.639206 & 1.378896 \\
\hline 8 & 0.086073 & 10.87785 & 1.813411 & 80.44752 & 2.632648 & 2.855900 & 1.372676 \\
\hline 9 & 0.086340 & 10.81862 & 1.897712 & 80.00313 & 2.736307 & 3.007158 & 1.537077 \\
\hline 10 & 0.086773 & 10.90877 & 1.894175 & 79.79490 & 2.744190 & 3.062776 & 1.595192 \\
\hline 11 & 0.086934 & 11.36290 & 1.880503 & 79.24981 & 2.724737 & 3.134925 & 1.647124 \\
\hline 12 & 0.087125 & 11.47555 & 1.884804 & 79.07314 & 2.713280 & 3.139069 & 1.714153 \\
\hline 13 & 0.087239 & 11.44816 & 1.909865 & 78.98569 & 2.780058 & 3.164750 & 1.711478 \\
\hline 14 & 0.087295 & 11.43043 & 1.919795 & 78.86287 & 2.905327 & 3.169055 & 1.712523 \\
\hline 15 & 0.087336 & 11.46768 & 1.920851 & 78.81851 & 2.906569 & 3.169968 & 1.716423 \\
\hline Ordenamiento de Cholesky: D(GIMP) D(GEXP) D(GGOB) D(GINV) D(GCONS) D(GPIB)
\end{tabular}

Fuente: Elaboración propia en base a los resultados del modelo VAR

Para analizar la relación de largo plazo se procedió a realizar el test de Johansen. Se verificó la existencia de un vector de cointegración en el caso de tendencia de datos lineal y con el tipo de test con intercepto y tendencia. Los resultados del test de Johansen se encuentran descritos a continuación:

\section{Cuadro 6 \\ Test de Johansen}

\begin{tabular}{lccccc}
\hline $\begin{array}{c}\text { Tendencia } \\
\text { de datos: }\end{array}$ & Ninguna & Ninguna & Lineal & Lineal & Cuadrática \\
\hline \multirow{2}{*}{ Tipo de test } & Sin intercepto & Intercepto & Intercepto & Intercepto & Intercepto \\
\cline { 2 - 6 } & Sin tendencia & Sin tendencia & Sin tendencia & Tendencia & Tendencia \\
\hline Trace & 2 & 2 & 2 & 1 & 2 \\
\hline Max-Eig & 2 & 1 & 0 & 0 & 0 \\
\hline
\end{tabular}

Fuente: Elaboración propia en base a datos del INE 
Posteriormente se procedió a estimar el modelo de Vectores Autorregresivos con corrección de Error (VEC)4 La relación de largo plazo entre el gasto y el Producto Interno Bruto permanece invariante. Para detectar la causalidad existente entre ambas variables se procedió a realizar el test de causalidad de Granger en el modelo VEC.

Para el primer test planteado, se obtuvieron los siguientes resultados:

Ho: El PIB no tiene efecto, en el sentido de Granger, en el gasto público.

$H a$ : El PIB tiene efecto, en el sentido de Granger, en el gasto público.

\begin{tabular}{lllll}
\multicolumn{4}{c}{ Cuadro 7 } \\
Test de causalidad de Granger \\
\hline \multicolumn{4}{c}{ Variable dependiente GGOB } \\
\hline Variables & Chi-2 & df & Prob. \\
\hline GPIB & 1.557455 & 4 & 0.8164 \\
\hline
\end{tabular}

Fuente: Elaboración propia en base a la los resultados del modelo VEC

Para el segundo test planteado, se obtuvieron los siguientes resultados:

Ho: El gasto público no tiene efecto, en el sentido de Granger, en el PIB.

$H a$ : EL gasto público tiene efecto, en el sentido de Granger, en el PIB.

\begin{tabular}{|c|c|c|c|}
\hline \multicolumn{4}{|c|}{$\begin{array}{c}\text { Cuadro } 8 \\
\text { Test de causalidad de Granger }\end{array}$} \\
\hline \multicolumn{4}{|c|}{ Variable dependiente GPIB } \\
\hline Variables & Chi-2 & df & Prob. \\
\hline GGOB & 1.861079 & 4 & 0.7613 \\
\hline
\end{tabular}

Fuente: Elaboración propia en base a los resultados del modelo VEC

Como podemos apreciar, ambos test rechazan la hipótesis nula de causalidad en el sentido de Granger. Este resultado nos muestra que ambas variables no se producen efectos entre sí en el largo plazo. Por lo tanto, si analizamos la relación de largo plazo de las variables, podemos concluir que se cumple la hipótesis de neutralidad. Esto se evidencia en el análisis de las funciones impulso-respuesta; como podemos apreciar a continuación, la relación de largo plazo entre ambas variables es prácticamente nula.

4 Los resultados del modelo VEC y las pruebas de estabilidad y auto-correlación serial se encuentran detallados en los Anexos 6 y 7 , respectivamente. 


\section{Gráfico 1: Funciones de impulso-respuesta Modelo VEC5}

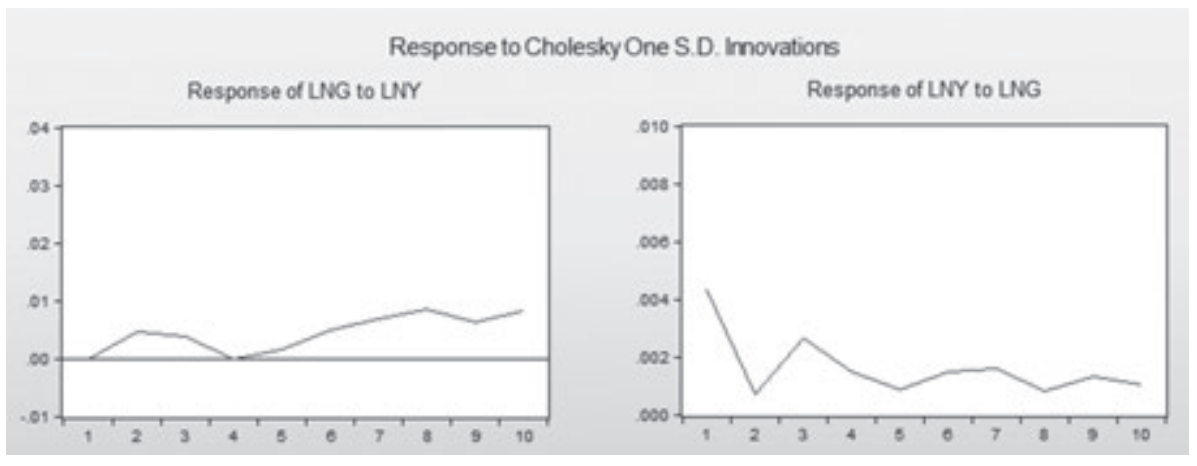

Fuente: elaboración propia en base a la los resultados del modelo VEC

\section{Conclusiones}

La teoría keynesiana y la ley de Wagner coinciden en la existencia de un alto nivel de correlación existente entre el Producto Interno Bruto y el gasto de Gobierno. Sin embargo, estas dos teorías se diferencian en la causalidad que, se asume, existe entre ambas variables. Por un lado, Wagner asegura que la causalidad entre ambas variables se manifiesta desde el Producto Interno Bruto hacia el gasto de Gobierno. Es decir, a medida que un país crece, acumula más recursos, logrando así incrementar el campo de acción del gobierno, teniendo este último, por tanto, una mayor cantidad de recursos disponibles.

Por otro lado, Keynes afirma que la causalidad entre estas variables sería la inversa, es decir que el gasto de Gobierno precedería al incremento del Producto Interno Bruto. Bajo este punto de vista, el gasto de Gobierno sería una herramienta fundamental para lograr un mayor crecimiento de la economía, y así incrementar los recursos de un país.

Si bien ambas teorías tienen un sólido sustento teórico, es importante averiguar cuál de estas dos teorías se cumple en la economía boliviana. Es por ello que este documento presenta evidencia empírica para el caso boliviano. Con la ayuda de un modelo de Vectores Autorregresivos, un modelo de Vectores con Corrección de Erroryla posterior determinación de la causalidad entre las variables de gasto de Gobierno y Producto Interno Bruto, se pudo evidenciar que el gasto de Gobierno tiene efecto, en el sentido de Granger, en el Producto

5 El impacto de las demás variables endógenas sobre el PIB se encuentra descrito en el Anexo 8. 
Interno Bruto en el corto plazo. Mientras que la causalidad de ambas variables en el largo plazo es nula.

Los hallazgos del documento demuestran que la ley de Wagner no se cumple en la economía boliviana, a diferencia de la teoría keynesiana, la cual sí se cumple. Este resultado demuestra que el gasto de Gobierno es una herramienta importante para el crecimiento de la economía. Sin embargo, la magnitud del multiplicador del gasto es negativa y poco significativa, por lo que la efectividad del mismo dependerá de la forma en que este gasto sea asignado por parte de las autoridades pertinentes.

Fecha de recepción: 18 de marzo de 2016. Fecha de aceptación: 27 de abril de 2016. Manejado por la A.B.C.E. 


\section{Referencias}

1. Arias, E. y C. Torres. 2004. "Modelos VAR y VECM para el pronostico de corto plazo de las importaciones de Costa Rica”. Departamento de Investigaciones Económicas, Documento de Trabajo N²2. Banco Central de Costa Rica.

2. Aschauer, D. A. 1989. "Is public expenditure productive?", Journal of Monetary economics, 23(2), 177-200.

3. Banco Central de Bolivia. 2008. Boletín Estadístico. Gerencia de Estudios Económicos. La Paz, Bolivia.

4. Cheng, B. S., y T. W. Lai. 1997. "Government expenditures and economic growth in South Korea: A VAR approach”, Journal of Economic Development, 22(1), 11-24.

5. Colmes, M. 1996). "Presupuesto por resultados". Revista Internacional de Presupuestos Públicos, $\mathrm{N}^{\circ} 30$.

6. Comin, F., D. Diaz y J. Revuelta. 2009. “La relación entre el crecimiento económico y el gasto público en Argentina, Brasil, España y México durante el siglo XX”. En: XVI Encuentro de Economía Pública, 5 y 6 de febrero de 2009, Palacio de Congresos de Granada (p.125).

7. Dogan, E. y T. C. Tang. 2011. "Government expenditure and national income: Causality tests for five South East Asian countries”, International Business \& Economics Research Journal (IBER), 5(10).

8. Dornbusch, R. y S. Edwards. 1990. "La macroeconomía del populismo en América Latina”, Trimestre Económico, Fondo de Cultura Económica. 121-162.

9. Dornbusch, R. y S. Fischer. 1992.”Macroeconomáa. México: Mc.Graw Hill, quinta edición.

10. Ebaidalla, E. M. 2013. "Causality between Government Expenditure and National Income: Evidence from Sudan”, Journal of Economic Cooperation and Development, 34(4), 61-76.

11. Enders, W. 2008. Applied econometric time series. John Wiley \& Sons.

12. Fundación Milenio. 2013. "Informe de Milenio sobre la economía, 2006-2013".

13. Humérez,J.y F.Jiménez. 1995. "El impacto de los flujos de capital y la política monetaria: el caso de Bolivia”, Análisis Económico, 12, 191-222.

14. Islam, A. M. 2001. "Wagner's law revisited: cointegration and exogeneity tests for the USA". Applied Economics Letters, 8(8), 509-515.

15. Johansen, S. y K. Juselius. 1990. "Maximum likelihood estimation and inference on cointegration with applications to the demand for money", Oxford Bulletin of Economics and Statistics, 52(2), 169-210. 
16. Keynes, J. M. 1936. The general theory of interest, employment and money. London: Mcmillan.

17. Magazzino, C. 2012. "Wagner versus Keynes: Public spending and national income in Italy", Journal of Policy Modeling, 34(6), 890-905.

18. McCandless, G., M. F. Gabrielli y T. Murphy 2001. "Modelos econométricos de predicción macroeconómica en la Argentina”. Banco Central de la República Argentina, Documento de Trabajo $\mathrm{N}^{\circ} 19$.

19. Molina Diaz, G. 2014. "Cuantificación de los ingresos y gastos del sector publico no financiero, entre 1998 y 2013". En: Juan Antonio Morales (ed.), ¿Dónde está la plata? Los ingresos extraordinarios de la bonanza 2006-2013, 47-58, Fundación Milenio.

20. ------- 1992. "El ajuste estructural de la economía boliviana y el proceso de reforma presupuestaria”. Ponencia presentada a la Reunión Anual de la Asociación Interamericana e Ibérica de Presupuesto Público.

21. ------ 1999. "El marco normativo y conceptual del sistema presupuestario de Bolivia”. Ministerio de Hacienda, Viceministerio de Presupuesto y Contaduría, mimeografiado.

22. - -----1999. "La gestión por resultados, asignación racional de recursos, la técnica del presupuesto en base cero y el presupuesto público para la gestión fiscal del 2000”. Documento preparado para el seminario interno del Ministerio de Hacienda, La Paz, 5 de noviembre de 1999.

23. ----- 2001. "Análisis comparativo de los sistemas de presupuesto de los países latinoamericanos". Documento mimeografiado presentado en el "V Curso de presupuesto, contabilidad y control del gasto público". Instituto de Estudios Fiscales Ministerio de Hacienda de España, realizado en la ciudad de Madrid, España.

24. ------ 2001. "El diseño de políticas públicas en Bolivia: algunas consideraciones al respecto". Documento presentado en la "I Conferencia Internacional sobre los poderes ejecutivo y legislativo respecto al tratamiento sobre los sistemas de administración financiera", organizado por Fundación Alemana para el Desarrollo, Asociación Internacional de Presupuesto Público y Ministerio de Hacienda de la República del Perú.

25. ------- 2002. "Alternativas de financiamiento para la infraestructura". Documento mimeografiado presentado en el "XXIX Seminario internacional de presupuesto público”, realizado en la ciudad de México.

26. ------- 2003. "El sistema presupuestario boliviano 1960-2003 y el presupuesto plurianual". Documento mimeografiado, Ministerio de Hacienda.

27. -------- 2003. "Vinculación Plan de Gobierno y presupuesto en Bolivia". Documento mimeografiado, Ministerio de Hacienda. 
28. -----2005. “La hacienda pública de Bolivia”, Temas en la crisis.

29. - ----- 2006. "Evaluación de los sistemas integrados de administración financiera en Bolivia”. Documento mimeografiado presentado en el "XXXIII Seminario Internacional de Presupuesto Público”, realizado en Asunción, Paraguay, por la Asociación Paraguaya de Presupuesto Público y Ministerio de Hacienda.

30. ------- 2007. "La relación entre los poderes ejecutivo y legislativo en el tratamiento del presupuesto público: el caso de Bolivia". Documento presentado en el "XXXIV Seminario Internacional de Presupuesto Público”, realizado en la ciudad de Panamá, República de Panamá, por la Asociación Panameña de Presupuesto Público, Ministerio de Hacienda y ASIP.

31. -------- 2011. "El presupuesto público plurianual participativo: una propuesta de implementación para Bolivia”, FUNDAPPAC.

32. Montero Kuscevic, C. M. 2012. "Inversión pública en Bolivia y su incidencia en el crecimiento económico: un análisis desde la perspectiva espacial”. Revista de Análisis del Banco Central de Bolivia, № 31 .

33. Moscoso, G. M. y F. S. Laserna. 2012. "Bolivia: efectos dinámicos y efectividad de la política fiscal". En: Fernando Candia y otros, Gasto fiscal y bien común, 1-30, Fundación Milenio.

34. Musgrave, R. A. y A. T. Peacock (eds.). 1967. Classics in the Theory of Public Finance. London: Macmillan.

35. Puig, J. P. 2015. "Multiplicador del gasto público en Bolivia: una primera aproximación”, Revista Latinoamericana de Desarrollo Económico, (24), 47-78.

36. Richter, C. y D. Paparas. 2012. The Validity of Wagner's Law in Greece during the last 2 centuries, Internacional Network for Economic Research.

37. Sachs, J. y F. Larrain. 1994. Macroeconomía en la economía global. México: Prentice Hall Hispanoamerica S.A, primera edición en español.

38. Sims, C. A. 1980. "Macroeconomics and reality", Econométrica, Journal of the Econometric Society, 1-48.

39. Tanzi Vito y H. Zee Howell. 2008. "Política fiscal y crecimiento a largo plazo". ASIP, N 38.

40. Wagner, A. 1883. "Three extracts on public finance". En: R. Musgrave y A. Peacock (eds.) Classics in the Theory of Public Finance. London: Macmillan. 


\section{Anexos}

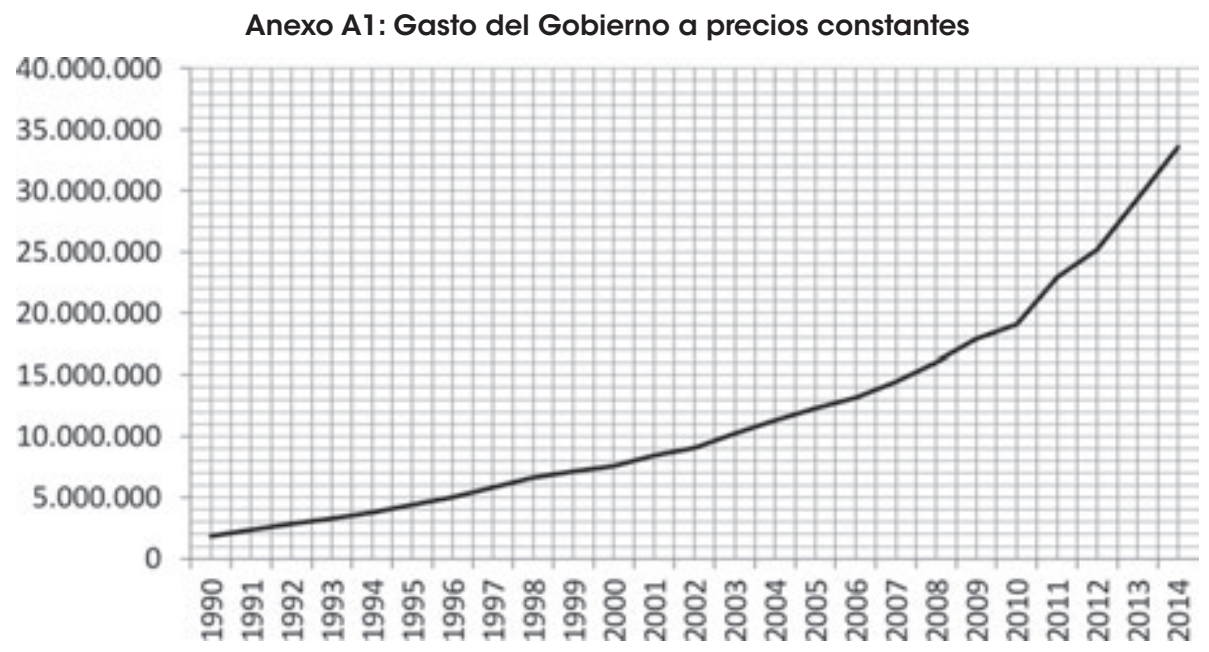

Fuente: elaboración propia en base a datos del INE

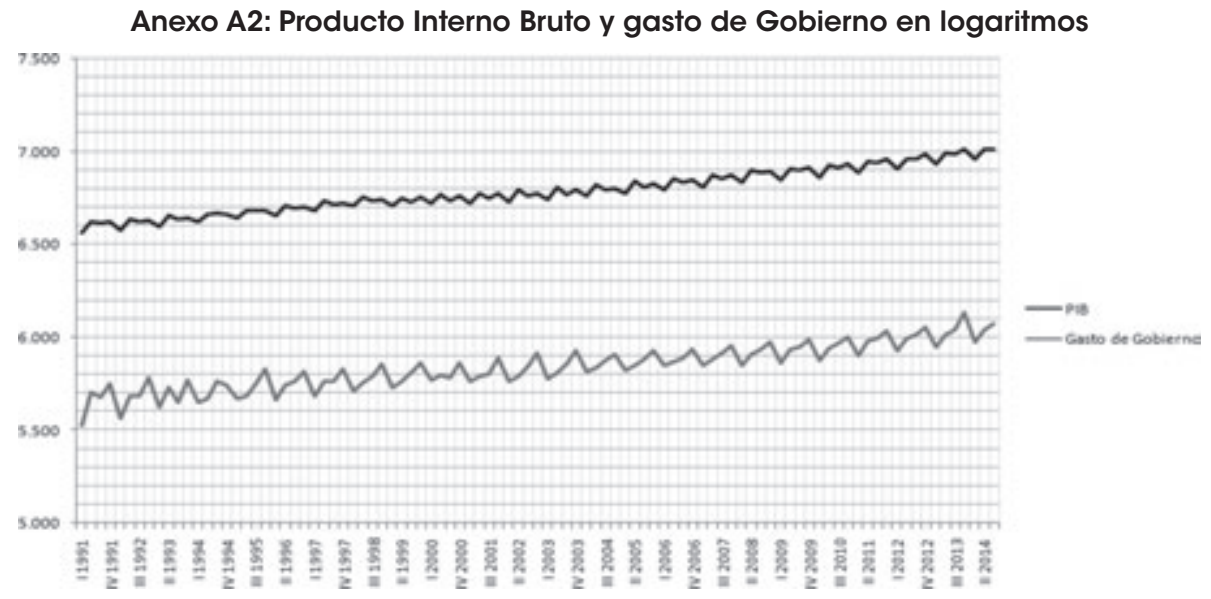

Fuente: elaboración propia en base a datos del INE 


\section{Anexo A3: Resultado del Modelo de Vectores Autorregresivos (VAR)}

\begin{tabular}{|c|c|c|c|c|c|c|}
\hline & D(GIMP) & $D(G E X P)$ & $D(G G O B)$ & $D(G I N V)$ & $D$ (GCONS) & $D(G P I B)$ \\
\hline \multirow[t]{3}{*}{$\mathrm{D}(\mathrm{GIMP}(-1))$} & -0.046320 & 0.801648 & 0.123102 & -0.151535 & -0.006774 & 0.021656 \\
\hline & $(0.25433)$ & (0.22033) & (0.14937) & $(0.60438)$ & $(0.04213)$ & (0.03615) \\
\hline & {$[-0.18213]$} & [ 3.63845$]$ & [ 0.82416$]$ & {$[-0.25073]$} & {$[-0.16078]$} & [ 0.59902] \\
\hline \multirow[t]{3}{*}{$\mathrm{D}(\mathrm{GIMP}(-2))$} & 0.310028 & 0.399437 & 0.131034 & 0.996426 & 0.033151 & 0.031639 \\
\hline & $(0.29410)$ & $(0.25478)$ & $(0.17272)$ & (0.69889) & $(0.04872)$ & $(0.04181)$ \\
\hline & [ 1.05415$]$ & [ 1.56777$]$ & [ 0.75863] & [ 1.42572] & [ 0.68049] & [ 0.75680$]$ \\
\hline \multirow[t]{3}{*}{$\mathrm{D}(\mathrm{GIMP}(-3))$} & 0.372746 & 0.381458 & 0.252853 & 0.907528 & 0.054589 & 0.035143 \\
\hline & (0.28950) & $(0.25079)$ & (0.17002) & $(0.68795)$ & (0.04795) & $(0.04115)$ \\
\hline & [ 1.28756$]$ & [ 1.52102$]$ & [ 1.48720$]$ & [ 1.31918$]$ & [ 1.13835$]$ & [ 0.85399] \\
\hline \multirow[t]{3}{*}{$\mathrm{D}(\mathrm{GIMP}(-4))$} & 0.191303 & -0.079666 & -0.008685 & 0.524650 & -0.048390 & -0.039747 \\
\hline & $(0.24974)$ & $(0.21635)$ & $(0.14667)$ & $(0.59348)$ & $(0.04137)$ & $(0.03550)$ \\
\hline & {$[0.76600]$} & {$[-0.36823]$} & {$[-0.05921]$} & [ 0.88403] & [-1.16971] & {$[-1.11962]$} \\
\hline \multirow[t]{3}{*}{$\mathrm{D}(\operatorname{GEXP}(-1))$} & -0.321600 & -0.911826 & -0.126449 & -0.153316 & -0.029469 & -0.005340 \\
\hline & (0.27229) & $(0.23588)$ & (0.15991) & $(0.64705)$ & $(0.04510)$ & $(0.03871)$ \\
\hline & {$[-1.18111]$} & {$[-3.86563]$} & {$[-0.79074]$} & {$[-0.23695]$} & {$[-0.65337]$} & {$[-0.13796]$} \\
\hline \multirow[t]{3}{*}{$\mathrm{D}(\mathrm{GEXP}(-2))$} & -0.537102 & -0.808609 & -0.305762 & -1.045208 & -0.038242 & -0.070075 \\
\hline & $(0.30360)$ & $(0.26301)$ & $(0.17830)$ & $(0.72147)$ & (0.05029) & $(0.04316)$ \\
\hline & {$[-1.76910]$} & {$[-3.07444]$} & {$[-1.71484]$} & {$[-1.44872]$} & {$[-0.76042]$} & {$[-1.62372]$} \\
\hline \multirow[t]{3}{*}{$\mathrm{D}(\operatorname{GEXP}(-3))$} & -0.952689 & -0.544312 & -0.205537 & -1.651322 & -0.060461 & -0.028110 \\
\hline & (0.32022) & $(0.27741)$ & (0.18806) & $(0.76096)$ & (0.05304) & $(0.04552)$ \\
\hline & {$[-2.97508]$} & {$[-1.96213]$} & {$[-1.09290]$} & {$[-2.17004]$} & [-1.13983] & {$[-0.61754]$} \\
\hline \multirow[t]{3}{*}{$D(\operatorname{GEXP}(-4))$} & -0.469030 & -0.193733 & -0.038261 & -0.586268 & 0.004906 & -0.001537 \\
\hline & $(0.25571)$ & $(0.22152)$ & $(0.15017)$ & $(0.60765)$ & $(0.04236)$ & $(0.03635)$ \\
\hline & {$[-1.83425]$} & {$[-0.87457]$} & {$[-0.25478]$} & {$[-0.96481]$} & [ 0.11584$]$ & {$[-0.04228]$} \\
\hline \multirow[t]{3}{*}{$\mathrm{D}(\mathrm{GGOB}(-1))$} & -0.356549 & -0.324006 & -0.989338 & -0.771558 & 0.001383 & -0.073003 \\
\hline & $(0.24527)$ & $(0.21248)$ & (0.14405) & $(0.58286)$ & (0.04063) & $(0.03487)$ \\
\hline & {$[-1.45367]$} & {$[-1.52487]$} & {$[-6.86812]$} & {$[-1.32375]$} & [ 0.03403] & {$[-2.09384]$} \\
\hline \multirow[t]{3}{*}{$\mathrm{D}(\mathrm{GGOB}(-2))$} & -0.661952 & -0.669175 & -0.652674 & -0.184632 & 0.004375 & -0.047421 \\
\hline & $(0.32031)$ & $(0.27748)$ & $(0.18811)$ & $(0.76116)$ & $(0.05306)$ & $(0.04553)$ \\
\hline & {$[-2.06663]$} & {$[-2.41161]$} & {$[-3.46958]$} & {$[-0.24257]$} & [ 0.08245$]$ & {$[-1.04149]$} \\
\hline \multirow[t]{3}{*}{$\mathrm{D}(\mathrm{GGOB}(-3))$} & -0.357071 & -0.134884 & -0.513683 & -0.770599 & -0.051646 & -0.061933 \\
\hline & $(0.30132)$ & (0.26103) & (0.17696) & $(0.71604)$ & (0.04991) & $(0.04283)$ \\
\hline & {$[-1.18503]$} & {$[-0.51674]$} & {$[-2.90280]$} & {$[-1.07620]$} & {$[-1.03474]$} & {$[-1.44593]$} \\
\hline
\end{tabular}




\begin{tabular}{|c|c|c|c|c|c|c|}
\hline \multirow[t]{3}{*}{$\mathrm{D}(\mathrm{GGOB}(-4))$} & -0.381520 & -0.195791 & -0.377663 & -0.500572 & -0.031858 & -0.071176 \\
\hline & $(0.25318)$ & $(0.21933)$ & $(0.14869)$ & $(0.60165)$ & $(0.04194)$ & $(0.03599)$ \\
\hline & {$[-1.50691]$} & {$[-0.89268]$} & [-2.53992] & {$[-0.83200]$} & {$[-0.75963]$} & {$[-1.97767]$} \\
\hline \multirow[t]{3}{*}{$D(\operatorname{GINV}(-1))$} & -0.203734 & -0.351681 & -0.029454 & -0.580359 & -0.000408 & -0.014938 \\
\hline & $(0.12077)$ & (0.10462) & $(0.07093)$ & (0.28699) & $(0.02001)$ & (0.01717) \\
\hline & {$[-1.68697]$} & {$[-3.36143]$} & {$[-0.41527]$} & {$[-2.02222]$} & {$[-0.02040]$} & {$[-0.87017]$} \\
\hline \multirow[t]{3}{*}{$\mathrm{D}(\mathrm{GINV}(-2))$} & -0.254084 & -0.261239 & -0.067032 & -0.812950 & -0.006544 & -0.032538 \\
\hline & $(0.14018)$ & $(0.12144)$ & $(0.08233)$ & $(0.33313)$ & $(0.02322)$ & (0.01993) \\
\hline & {$[-1.81250]$} & {$[-2.15115]$} & {$[-0.81420]$} & {$[-2.44035]$} & {$[-0.28183]$} & {$[-1.63284]$} \\
\hline \multirow[t]{3}{*}{$D(\operatorname{GINV}(-3))$} & -0.361971 & -0.250200 & -0.089928 & -0.851683 & -0.022260 & -0.024490 \\
\hline & $(0.14496)$ & $(0.12558)$ & $(0.08513)$ & $(0.34448)$ & $(0.02401)$ & $(0.02061)$ \\
\hline & {$[-2.49704]$} & {$[-1.99238]$} & {$[-1.05631]$} & {$[-2.47240]$} & {$[-0.92703]$} & {$[-1.18851]$} \\
\hline \multirow[t]{3}{*}{$\mathrm{D}(\mathrm{GINV}(-4))$} & -0.261255 & -0.069383 & -0.023450 & -0.416847 & 0.009100 & 0.010177 \\
\hline & $(0.12376)$ & $(0.10722)$ & $(0.07269)$ & $(0.29411)$ & $(0.02050)$ & $(0.01759)$ \\
\hline & [-2.11090] & {$[-0.64712]$} & {$[-0.32261]$} & {$[-1.41732]$} & [ 0.44389] & [ 0.57849] \\
\hline \multirow[t]{3}{*}{$\mathrm{D}(\mathrm{GCONS}(-1))$} & -1.156215 & -2.368869 & -1.256225 & 0.420260 & -0.685720 & -0.341623 \\
\hline & (1.03653) & $(0.89795)$ & $(0.60875)$ & $(2.46317)$ & (0.17170) & $(0.14734)$ \\
\hline & {$[-1.11547]$} & {$[-2.63810]$} & {$[-2.06363]$} & {$[0.17062]$} & {$[-3.99376]$} & {$[-2.31856]$} \\
\hline \multirow[t]{3}{*}{$\mathrm{D}(\mathrm{GCONS}(-2))$} & -1.583379 & -2.121983 & -1.195191 & -1.102930 & -0.442259 & -0.301673 \\
\hline & (1.17987) & $(1.02212)$ & $(0.69293)$ & $(2.80380)$ & (0.19544) & $(0.16772)$ \\
\hline & [-1.34199] & {$[-2.07606]$} & {$[-1.72484]$} & {$[-0.39337]$} & {$[-2.26287]$} & {$[-1.79869]$} \\
\hline \multirow[t]{3}{*}{$D(\operatorname{GCONS}(-3))$} & -1.654093 & -1.909053 & -0.362832 & -1.499264 & -0.401680 & -0.109198 \\
\hline & (1.18349) & $(1.02525)$ & (0.69505) & (2.81239) & (0.19604) & $(0.16823)$ \\
\hline & {$[-1.39764]$} & {$[-1.86203]$} & {$[-0.52202]$} & {$[-0.53309]$} & {$[-2.04896]$} & {$[-0.64909]$} \\
\hline \multirow[t]{3}{*}{$\mathrm{D}(\mathrm{GCONS}(-4))$} & -1.668607 & -0.484539 & 0.170865 & 0.531683 & -0.280010 & 0.095183 \\
\hline & $(0.97306)$ & $(0.84296)$ & $(0.57147)$ & $(2.31234)$ & $(0.16118)$ & $(0.13832)$ \\
\hline & {$[-1.71480]$} & {$[-0.57480]$} & [ 0.29899] & [ 0.22993] & {$[-1.73720]$} & [ 0.68813] \\
\hline \multirow[t]{3}{*}{$D(G P I B(-1))$} & 2.526215 & 1.800632 & 0.896886 & 3.792232 & 0.655112 & 0.088438 \\
\hline & $(1.20805)$ & (1.04653) & $(0.70948)$ & $(2.87076)$ & $(0.20011)$ & $(0.17172)$ \\
\hline & [ 2.09115$]$ & [ 1.72057$]$ & [ 1.26415$]$ & [ 1.32099] & [ 3.27377$]$ & [ 0.51500$]$ \\
\hline \multirow[t]{3}{*}{$\mathrm{D}(\mathrm{GPIB}(-2))$} & 4.926171 & 3.850094 & 1.289798 & 5.180233 & 0.401692 & 0.454323 \\
\hline & $(1.44980)$ & $(1.25596)$ & $(0.85145)$ & $(3.44523)$ & $(0.24015)$ & $(0.20609)$ \\
\hline & [ 3.39784$]$ & [ 3.06547] & [ 1.51482$]$ & [ 1.50360$]$ & [ 1.67265$]$ & [ 2.20451] \\
\hline
\end{tabular}




\begin{tabular}{lrrrrrr}
\hline D(GPIB(-3)) & 3.799347 & 1.327178 & 0.555896 & 9.039339 & 0.475245 & 0.349853 \\
& $(1.52945)$ & $(1.32496)$ & $(0.89823)$ & $(3.63451)$ & $(0.25335)$ & $(0.21741)$ \\
& {$[2.48413]$} & {$[1.00168]$} & {$[0.61888]$} & {$[2.48709]$} & {$[1.87586]$} & {$[1.60918]$} \\
\hline D(GPIB(-4)) & 2.315819 & 0.370620 & 0.196304 & 3.614932 & 0.447819 & 0.158073 \\
& $(1.38081)$ & $(1.19619)$ & $(0.81094)$ & $(3.28129)$ & $(0.22873)$ & $(0.19628)$ \\
& {$[1.67715]$} & {$[0.30983]$} & {$[0.24207]$} & {$[1.10168]$} & {$[1.95788]$} & {$[0.80534]$} \\
\hline C & -0.012076 & 0.043729 & 0.036049 & -0.110264 & 0.008337 & 0.009725 \\
& $(0.02328)$ & $(0.02017)$ & $(0.01367)$ & $(0.05533)$ & $(0.00386)$ & $(0.00331)$ \\
& {$[-0.51862]$} & {$[2.16789]$} & {$[2.63617]$} & {$[-1.99276]$} & {$[2.16154]$} & {$[2.93815]$} \\
\hline R-squared & 0.431022 & 0.456870 & 0.646397 & 0.429127 & 0.541553 & 0.402616 \\
Adj. R-squared & 0.235944 & 0.270655 & 0.525162 & 0.233399 & 0.384371 & 0.197798 \\
Sum sq. resids & 0.317174 & 0.238030 & 0.109397 & 1.791101 & 0.008703 & 0.006409 \\
S.E. equation & 0.067313 & 0.058313 & 0.039532 & 0.159960 & 0.011150 & 0.009569 \\
F-statistic & 2.209485 & 2.453446 & 5.331759 & 2.192464 & 3.445385 & 1.965728 \\
Log likelihood & 136.0544 & 149.6895 & 186.6166 & 53.82555 & 306.8549 & 321.3875 \\
Akaike AlC & -2.337988 & -2.625041 & -3.402455 & -0.606854 & -5.933788 & -6.239737 \\
Schwarz SC & -1.665915 & -1.952968 & -2.730382 & 0.065219 & -5.261715 & -5.567664 \\
Mean & 0.014732 & 0.014125 & 0.011616 & 0.013229 & 0.009439 & 0.010122 \\
dependent & 0.077008 & 0.068281 & 0.057370 & 0.182695 & 0.014211 & 0.010683 \\
S.D. dependent & 0.0196
\end{tabular}

Fuente: elaboración propia en base a datos del INE

\section{Anexo A4: Pruebas de estabilidad y auto-correlación serial}

Imverse Roots of AR Characteristic Polynomial

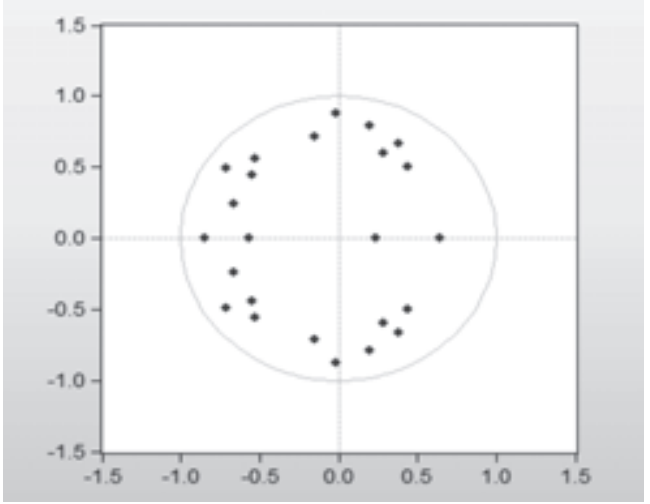

Fuente: elaboración propia en base a los resultados del modelo VAR 
Hipótesis nula: no existe auto-correlación serial.

\begin{tabular}{cll}
\hline Lags & LM-Stat & Prob \\
\hline 1 & 29.98545 & 0.7495 \\
\hline 2 & 39.28523 & 0.3249 \\
\hline 3 & 34.83132 & 0.5241 \\
\hline 4 & 31.96270 & 0.6611 \\
\hline 5 & 39.15251 & 0.3302 \\
\hline 6 & 40.12021 & 0.2925
\end{tabular}

Fuente: elaboración propia en base a los resultados del modelo VAR

Anexo A5: Funciones impulso-respuesta del Modelo de Vectores Autorregresivos VAR (variable de respuesta PIB)

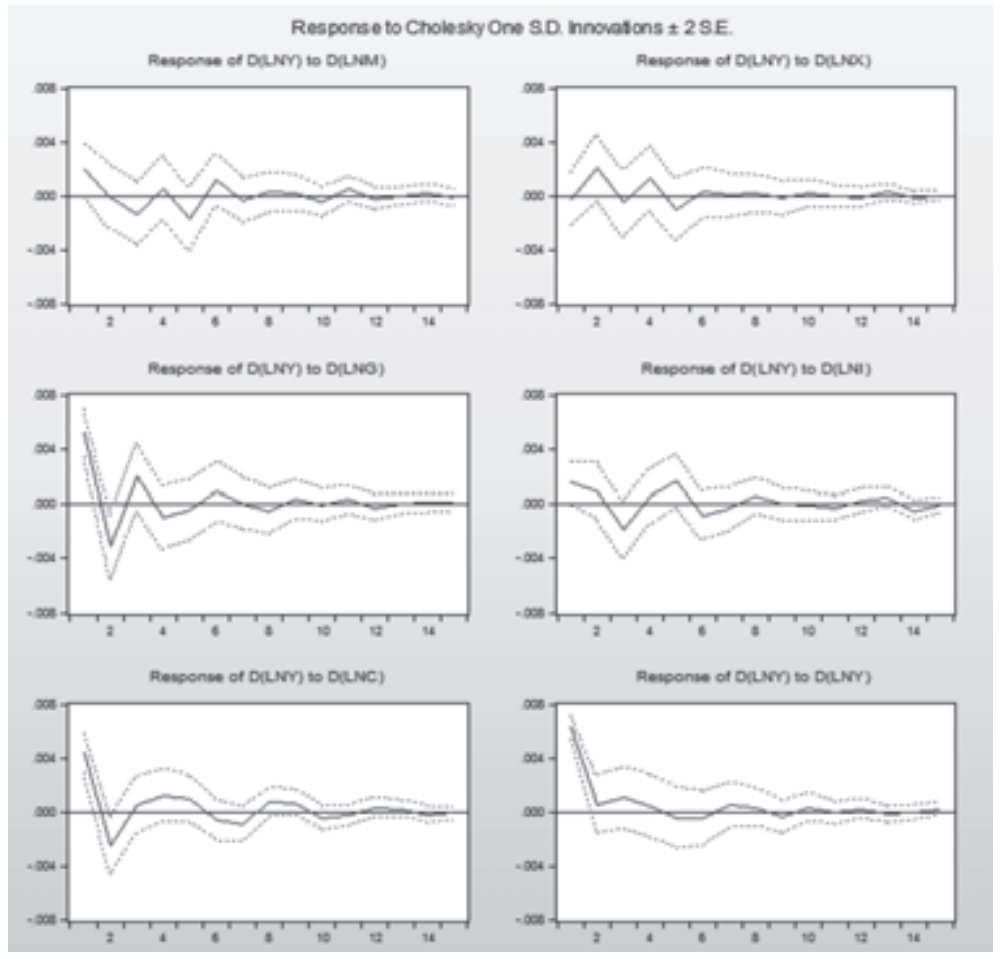

Fuente: elaboración propia en base a los resultados del modelo VAR 
Anexo A6: Resultado del Modelo de Vectores con Corrección de Error (VEC)

\begin{tabular}{|c|c|c|c|c|c|c|}
\hline $\begin{array}{l}\text { Cointegrating } \\
\text { Eq: }\end{array}$ & CointEq 1 & & & & & \\
\hline $\operatorname{GIMP}(-1)$ & 1.000000 & & & & & \\
\hline \multirow[t]{3}{*}{$\operatorname{GEXP}(-1)$} & 0.728677 & & & & & \\
\hline & $(0.48289)$ & & & & & \\
\hline & [ 1.50900$]$ & & & & & \\
\hline \multirow[t]{3}{*}{ GGOB(-1) } & -4.527877 & & & & & \\
\hline & $(1.34418)$ & & & & & \\
\hline & {$[-3.36851]$} & & & & & \\
\hline \multirow[t]{3}{*}{$\operatorname{GINV}(-1)$} & -0.177819 & & & & & \\
\hline & $(0.24585)$ & & & & & \\
\hline & {$[-0.72329]$} & & & & & \\
\hline \multirow[t]{3}{*}{ GCONS(-1) } & 1.466423 & & & & & \\
\hline & (2.97609) & & & & & \\
\hline & {$[0.49273]$} & & & & & \\
\hline \multirow[t]{3}{*}{ GPIB(-1) } & 4.278081 & & & & & \\
\hline & $(2.97706)$ & & & & & \\
\hline & [ 1.43702] & & & & & \\
\hline \multirow[t]{3}{*}{ @TREND(90Q1) } & -0.031266 & & & & & \\
\hline & $(0.01201)$ & & & & & \\
\hline & {$[-2.60403]$} & & & & & \\
\hline C & -49.21214 & & & & & \\
\hline Error Correction: & $\mathrm{D}(\mathrm{GIMP})$ & $D(G E X P)$ & $D(G G O B)$ & $D(G I N V)$ & D(GCONS) & $D(G P I B)$ \\
\hline \multirow[t]{3}{*}{ CointEq1 } & -0.176577 & -0.183373 & 0.129941 & -0.114959 & 0.027173 & 0.019137 \\
\hline & $(0.06677)$ & $(0.05655)$ & $(0.03806)$ & $(0.16594)$ & $(0.01114)$ & $(0.00969)$ \\
\hline & {$[-2.64459]$} & {$[-3.24289]$} & [ 3.41381$]$ & {$[-0.69279]$} & {$[2.44002]$} & [ 1.97477] \\
\hline \multirow[t]{3}{*}{$\mathrm{D}(\operatorname{GIMP}(-1))$} & -0.030009 & 0.818587 & 0.111098 & -0.140916 & -0.009284 & 0.019889 \\
\hline & $(0.24417)$ & $(0.20679)$ & $(0.13920)$ & $(0.60683)$ & $(0.04073)$ & $(0.03544)$ \\
\hline & {$[-0.12290]$} & [ 3.95857] & {$[0.79814]$} & {$[-0.23221]$} & {$[-0.22796]$} & [0.56122] \\
\hline \multirow[t]{3}{*}{$\mathrm{D}(\mathrm{GIMP}(-2))$} & 0.336362 & 0.426785 & 0.111655 & 1.013571 & 0.029099 & 0.028785 \\
\hline & $(0.28244)$ & $(0.23920)$ & $(0.16101)$ & (0.70194) & $(0.04711)$ & (0.04099) \\
\hline & [ 1.19091$]$ & [ 1.78423$]$ & {$[0.69346]$} & [ 1.44396$]$ & [ 0.61769$]$ & {$[0.70222]$} \\
\hline \multirow[t]{3}{*}{$\mathrm{D}(\operatorname{GIMP}(-3))$} & 0.417456 & 0.427889 & 0.219952 & 0.936637 & 0.047708 & 0.030298 \\
\hline & $(0.27836)$ & $(0.23574)$ & (0.15869) & $(0.69180)$ & $(0.04643)$ & $(0.04040)$ \\
\hline & [ 1.49969] & [ 1.81508$]$ & [ 1.38608$]$ & [ 1.35392$]$ & [ 1.02757$]$ & [ 0.74995] \\
\hline \multirow[t]{3}{*}{$\mathrm{D}(\mathrm{GIMP}(-4))$} & 0.200622 & -0.069988 & -0.015543 & 0.530717 & -0.049824 & -0.040757 \\
\hline & $(0.23972)$ & $(0.20301)$ & $(0.13666)$ & $(0.59576)$ & (0.03998) & (0.03479) \\
\hline & {$[0.83691]$} & {$[-0.34475]$} & {$[-0.11374]$} & [0.89083] & [-1.24613] & {$[-1.17148]$} \\
\hline
\end{tabular}




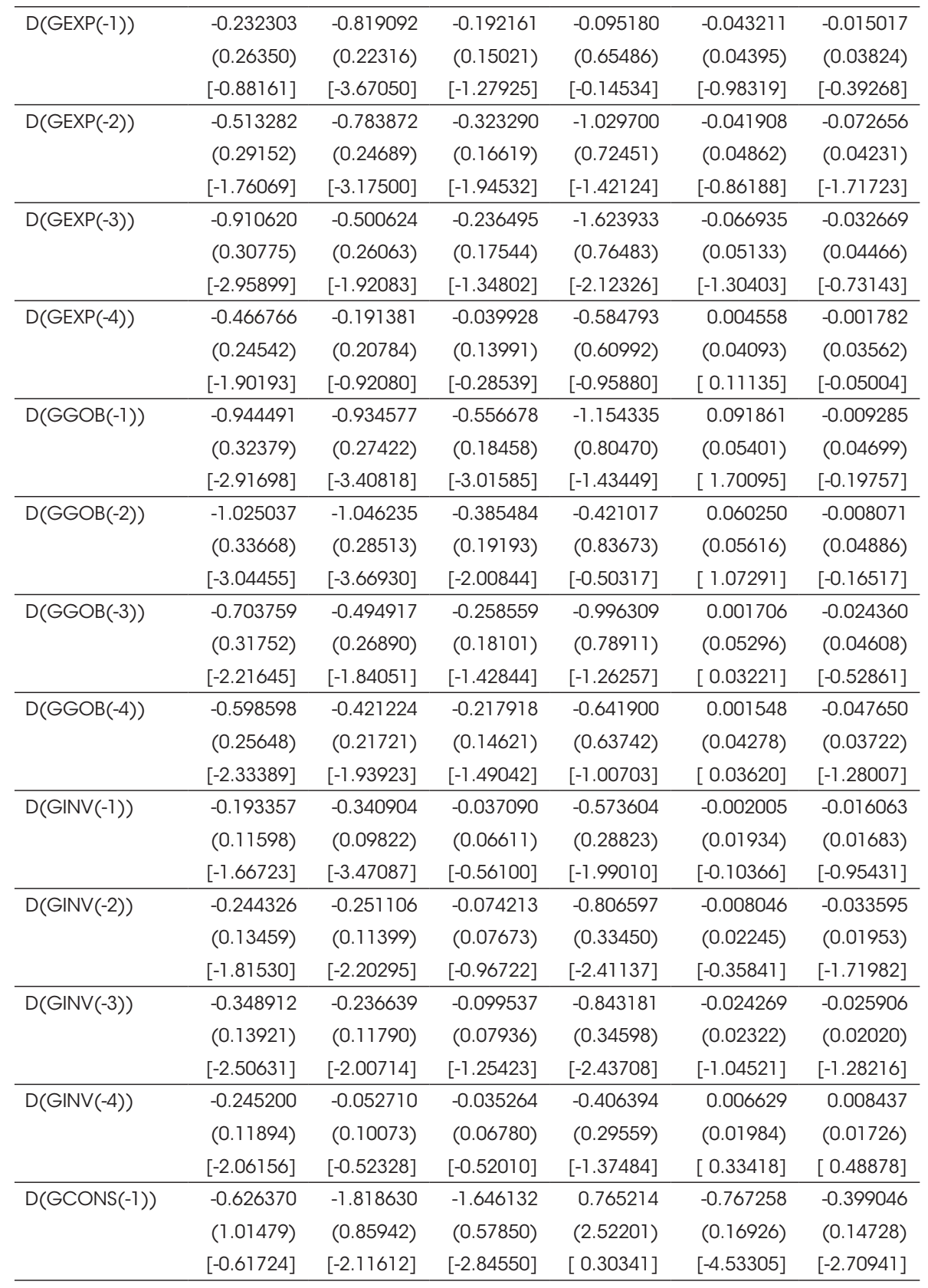




\begin{tabular}{|c|c|c|c|c|c|c|}
\hline \multirow[t]{3}{*}{$\mathrm{D}(\mathrm{GCONS}(-2))$} & -0.597767 & -1.098435 & -1.920492 & -0.461251 & -0.593935 & -0.408489 \\
\hline & $(1.19214)$ & $(1.00962)$ & $(0.67961)$ & (2.96277) & $(0.19884)$ & $(0.17302)$ \\
\hline & {$[-0.50142]$} & {$[-1.08797]$} & {$[-2.82589]$} & {$[-0.15568]$} & {$[-2.98701]$} & {$[-2.36092]$} \\
\hline \multirow[t]{3}{*}{$\mathrm{D}(\mathrm{GCONS}(-3))$} & -0.696275 & -0.914369 & -1.067679 & -0.875681 & -0.549079 & -0.213002 \\
\hline & (1.19220) & $(1.00967)$ & $(0.67964)$ & (2.96293) & (0.19885) & $(0.17303)$ \\
\hline & {$[-0.58402]$} & {$[-0.90561]$} & {$[-1.57094]$} & {$[-0.29555]$} & {$[-2.76127]$} & {$[-1.23101]$} \\
\hline \multirow[t]{3}{*}{$\mathrm{D}(\mathrm{GCONS}(-4))$} & -1.041173 & 0.167044 & -0.290856 & 0.940172 & -0.376566 & 0.027185 \\
\hline & (0.96357) & $(0.81604)$ & $(0.54930)$ & (2.39471) & $(0.16072)$ & $(0.13985)$ \\
\hline & {$[-1.08054]$} & [ 0.20470$]$ & {$[-0.52950]$} & {$[0.39260]$} & {$[-2.34306]$} & [0.19439] \\
\hline \multirow[t]{3}{*}{$\mathrm{D}(\mathrm{GPIB}(-1))$} & 3.523505 & 2.836308 & 0.162992 & 4.441513 & 0.501639 & -0.019643 \\
\hline & $(1.21922)$ & (1.03255) & $(0.69504)$ & (3.03006) & $(0.20336)$ & $(0.17695)$ \\
\hline & [ 2.88997] & [ 2.74691] & {$[0.23451]$} & [ 1.46582] & [ 2.46681] & {$[-0.11101]$} \\
\hline \multirow[t]{3}{*}{$\mathrm{D}(\mathrm{GPIB}(-2))$} & 5.627258 & 4.578166 & 0.773876 & 5.636674 & 0.293802 & 0.378343 \\
\hline & $(1.41648)$ & (1.19960) & $(0.80749)$ & (3.52030) & $(0.23626)$ & $(0.20558)$ \\
\hline & [ 3.97272] & [ 3.81640$]$ & [ 0.95837$]$ & [ 1.60119$]$ & [ 1.24357$]$ & [ 1.84036] \\
\hline \multirow[t]{3}{*}{$\mathrm{D}(\mathrm{GPIB}(-3))$} & 4.536211 & 2.092403 & 0.013646 & 9.519071 & 0.361849 & 0.269995 \\
\hline & $(1.49410)$ & $(1.26534)$ & $(0.85175)$ & (3.71322) & $(0.24920)$ & $(0.21685)$ \\
\hline & [ 3.03608$]$ & [ 1.65362$]$ & [ 0.01602$]$ & [ 2.56356$]$ & [ 1.45202$]$ & [ 1.24510] \\
\hline \multirow[t]{3}{*}{$\mathrm{D}(\mathrm{GPIB}(-4))$} & 2.964039 & 1.043789 & -0.280714 & 4.036953 & 0.348064 & 0.087822 \\
\hline & $(1.34771)$ & $(1.14137)$ & $(0.76829)$ & $(3.34941)$ & $(0.22479)$ & $(0.19560)$ \\
\hline & [2.19931] & {$[0.91451]$} & {$[-0.36537]$} & [ 1.20527$]$ & [ 1.54842$]$ & [ 0.44899] \\
\hline \multirow[t]{3}{*}{$\mathrm{C}$} & -0.060965 & -0.007041 & 0.072026 & -0.142093 & 0.015861 & 0.015023 \\
\hline & $(0.02900)$ & $(0.02456)$ & $(0.01653)$ & $(0.07208)$ & $(0.00484)$ & $(0.00421)$ \\
\hline & {$[-2.10203]$} & {$[-0.28666]$} & [ 4.35633] & {$[-1.97136]$} & [ 3.27874$]$ & [ 3.56908] \\
\hline R-squared & 0.483386 & 0.528701 & 0.697491 & 0.433070 & 0.577968 & 0.434572 \\
\hline Adj. R-squared & 0.296207 & 0.357941 & 0.587886 & 0.227661 & 0.425058 & 0.229707 \\
\hline Sum sq. resids & 0.287984 & 0.206550 & 0.093590 & 1.778728 & 0.008012 & 0.006066 \\
\hline S.E. equation & 0.064604 & 0.054713 & 0.036829 & 0.160557 & 0.010775 & 0.009376 \\
\hline F-statistic & 2.582482 & 3.096158 & 6.363697 & 2.108327 & 3.779787 & 2.121259 \\
\hline Log likelihood & 140.6404 & 156.4276 & 194.0296 & 54.15481 & 310.7862 & 323.9990 \\
\hline Akaike AIC & -2.413482 & -2.745844 & -3.537466 & -0.592733 & -5.995500 & -6.273663 \\
\hline Schwarz SC & -1.714526 & -2.046889 & -2.838510 & 0.106223 & -5.296544 & -5.574707 \\
\hline $\begin{array}{l}\text { Mean } \\
\text { dependent }\end{array}$ & 0.014732 & 0.014125 & 0.011616 & 0.013229 & 0.009439 & 0.010122 \\
\hline S.D. dependent & 0.077008 & 0.068281 & 0.057370 & 0.182695 & 0.014211 & 0.010683 \\
\hline
\end{tabular}

Fuente: elaboración propia en base a datos del INE 


\section{Anexo A7: Pruebas de estabilidad y auto-correlación serial} Imverse Roots of AR Characteristic Polynomial

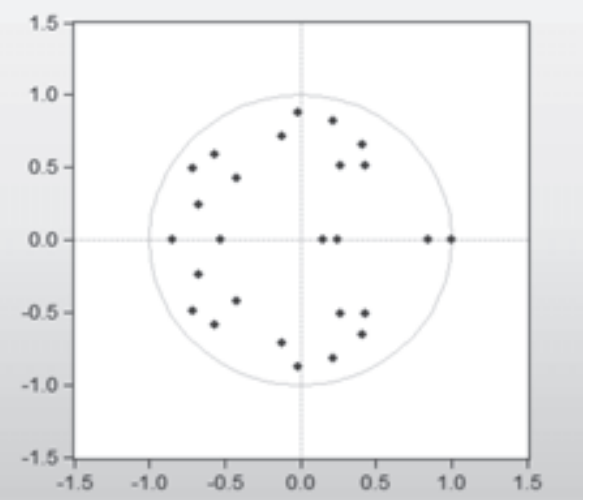

Fuente: elaboración propia en base a los resultados del modelo VAR

La especificación del VEC impone cinco raíces unitarias. Hipótesis nula: no existe auto-correlación serial.

\begin{tabular}{cll}
\hline Lags & LM-Stat & Prob \\
\hline 1 & 32.18732 & 0.6506 \\
\hline 2 & 37.47834 & 0.4012 \\
\hline 3 & 27.44273 & 0.8465 \\
\hline 4 & 32.60801 & 0.6307 \\
\hline 5 & 36.87439 & 0.4283 \\
\hline 6 & 42.74848 & 0.2038 \\
\hline
\end{tabular}

Fuente: elaboración propia en base a los resultados del modelo VAR 
Anexo A8: Funciones impulso-respuesta del Modelo VEC (variable de respuesta PIB)

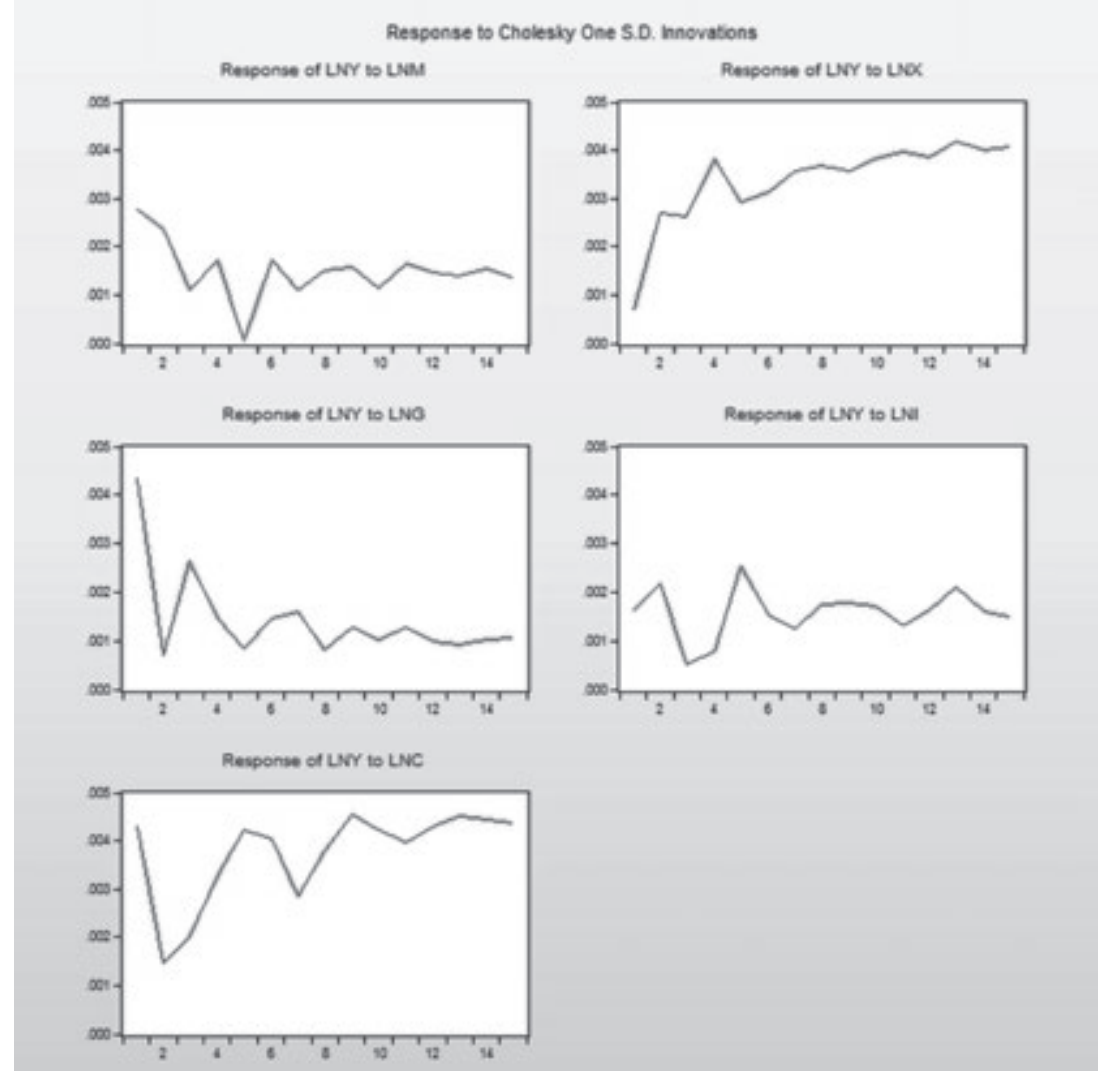

Fuente: elaboración propia en base a los resultados del modelo VAR 